\title{
Analysis of a 3D Unsteady Morphing Wing with Seamless Side-edge Transition
}

\author{
Chawki Abdessemed ${ }^{1}$, Yufeng Yao ${ }^{2}$, Abdessalem Bouferrouk ${ }^{3}$, Pritesh Narayan ${ }^{4}$ \\ Department of Engineering Design and Mathematics, University of the West of England, Coldharbour Lane, \\ Frenchay, Bristol BS16 1QY, United Kingdom.
}

In this paper, a comparative study between a NACA 0012 rectangular wing with a statically morphed Trailing Edge Flap (TEF) and the same wing with a hinged flap is performed at a Reynolds number based on the chord length of $\operatorname{Re}=0.62 \times 10^{6}$ and a Mach number of 0.115 . Furthermore, an unsteady flow analysis of a dynamically morphing wing is performed, taking care to model the flap side-edge between the morphing and static parts. The deformation is parametrized in time for the morphing TEF portion and a parametrization for the transition is introduced to eliminate the flap side-edge gap and model its deformation as a seamless surface. Dynamic meshing methods were used to deform the computational grid and accurately capture the aerodynamic features of the unsteady morphing wing. The modified parametrization method was implemented successfully and an analysis of the unsteady morphing effects was carried out. The Shear Stress Transport (SST) model was utilized to model turbulence in all studied configurations whose performances were evaluated for a range of angles of attack. It was found that at low Angles of Attack the morphing TEF with the seamless transition displays an increased aerodynamic efficiency compared with the hinged flap configuration, yet the performance of the morphed TEF deteriorates at higher AoA while the hinged flap wing performs consistently. Finally, this paper introduces a framework to model accurately a 3D morphing wing with a seamless transition using an unsteady parametrization method and dynamic meshing. The unsteady analysis of the dynamically morphed wing has shown that the TEF motion induces complex flow phenomena, paving the way for in-depth high fidelity analysis using the developed framework.

Nomenclature

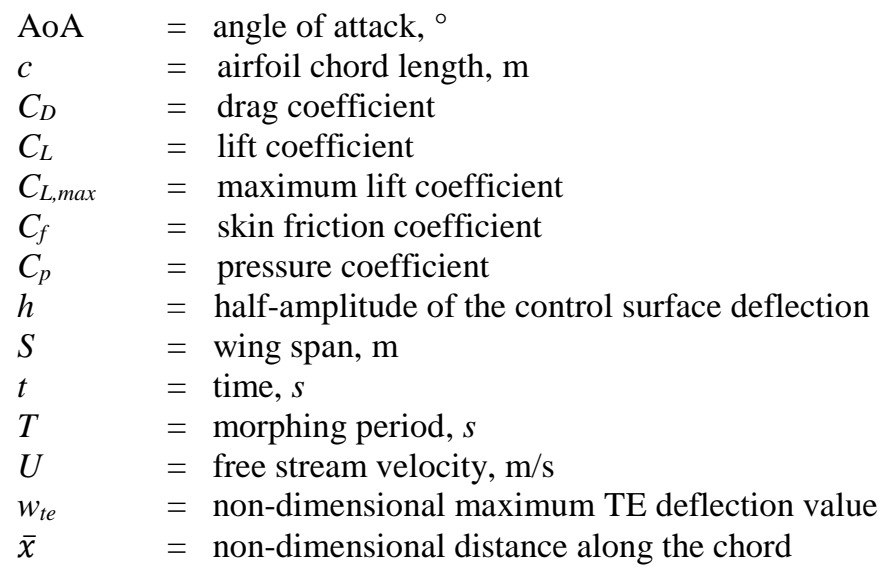

\footnotetext{
${ }^{1} \mathrm{PhD}$ student, Department of Engineering Design and Mathematics, AIAA Student member.

${ }^{2}$ Professor, Department of Engineering Design and Mathematics, AIAA Associate Fellow.

${ }^{3}$ Senior lecturer, Department of Engineering Design and Mathematics.

${ }^{4}$ Senior lecturer, Department of Engineering Design and Mathematics.
} 


$\begin{array}{ll}x_{s} & =\text { non-dimensional morphing start location } \\ y_{c} & =\text { non-dimensional camber line } \\ y_{t} & =\text { non-dimensional thickness distribution } \\ \bar{z} & =\text { non-dimensional spanwise transition distribution } \\ z_{t} & =\text { non-dimensional vertical TE displacement for the transition part }\end{array}$

\section{Introduction}

The aircraft industry has been under an increasing pressure to move toward greener and quieter aircraft through various frameworks such as the flightpath 2050 [1]. However, modern aircraft are reaching near peak levels of efficiency making the improvements of current technologies problematic, if not impossible. Therefore, engineers have been striving to re-imagine the present-day aircraft by employing cutting-edge technologies e.g. lightweight materials for the structures, unconventional fuselage configurations, highly efficient aircraft engines and adaptive structures for flight optimization and flow control; the latter being one of the challenges that researchers have focused on in the last decade. The benefits and challenges of morphing structures are well documented in literature and various reviews have been produced $[2,3]$. Given the recent advances in smart materials that reduce the weight and complexities of morphing systems, this advancement could make the large step increase in aerodynamic efficiency, and reduction of airframe noise through the application of morphing systems tangible.

A study conducted by NASA in its Elastically Shape Future Air Vehicle project [4, 5] showed that using morphing lifting surfaces for in-flight flow control resulted in drag reduction during cruise and enhanced lift performance during take-off and landing. Furthermore, a morphing Trailing Edge Flap (TEF) would seal the gaps present at the end of the control surfaces in both chord-wise and spanwise directions, eliminating the small pockets known for their high vorticity and significant source of airframe noise [6]. Several different approaches have been proposed to seal the flap side-edge such as the concept presented by Khorami et al. [7] where elastically deformable structures are introduced at each side-edge to passively deform with the flap and seal the gap. An additional concept, which has already reached the flight-test stage, is the one developed under NASA's Adaptive Compliant Trailing Edge (ACTE) project and FlexSys Inc.[8, 9]. The high-lift flaps of a Gulfstream III business jet were replaced by a morphing transition structure with a compliant fairing at the end of each flap to seal the gap (Fig. 1), with subsequent flight tests of this concept demonstrating that it is possible to reduce aircraft noise by as much as 30 percent [10]. However, this concept does not offer a smooth transition, which could still be a source of disturbance in the flow. Recently, Woods et al. [11] presented a design for a compliant morphing flap transition that offers a smooth transition, with an additional advantage being that the design can be integrated with the Fish Bone Active Camber (FishBAC) morphing airfoil [12].

On the numerical side, more work has been undertaken to quantify the efficiency of morphing concepts. Starting with optimization problems using low-fidelity panel-based methods as used by Molinari et al.[13] or the VortexLattice Methods (VLM) as used by Obradovic et al.[14], the Reynolds-Averaged Navier-Stokes (RANS) solver coupled with various turbulence models has also been applied for morphing wings applications, such as in Lyu $e t$ al.[15] where the Spalart-Allmaras model was used, up to 5\% drag reduction was reported. Ai et al.[16] studied an airfoil fitted with a morphing TEF, and found that the morphing TEF is more aerodynamically efficient at the angles of attack studied. The $k-\omega$-SST model was used by Woods et al. [17] for their analysis, and it was discovered that the start location affects the aerodynamic performance for all Reynold numbers investigated. Finally, Jawahar et al.[18] used the hybrid RANS-LES model Detached Eddy Simulation (DES) to study the aerodynamic and aeroacoustic flow around an airfoil with a rigid and morphing TEF, an increase of up to $13 \%$ in the $\mathrm{C}_{L, \max }$ was observed, but with a drag penalty that reduced the aerodynamic efficiency by $4 \%$. Initial qualitative results of a morphing concept with a seamless transition were performed by Woods et al. [12]. it was found that the use of this concept reduces significantly the pressure leakage from the lower to upper wing surface which results in an improvement of aerodynamic performance. This is further supported by a study ([19]) which compared the same morphing concept with a traditional unsealed configuration using both CFD and experimental methods, results showed that up to $18 \%$ gains in aerodynamic efficiency was achievable.

Most studies to date have simplified morphing problems to static morphing, thereby overlooking the dynamic effects that deforming motion of the TEF might have on the flow field or its contribution to the airframe noise. Abdessemed et al.[20, 21] introduced a framework to study dynamically morphing airfoils by modifying a parametrization method to include time and integrating it in the commercial software ANSYS Fluent [22] with the help of a User-Defined Function (UDF). The same methodology was used for a 2D aeroacoustic study of a harmonically morphing TEF [23]. Nevertheless, to capture the physics of real life morphing wings, the problem needs 
to be considered in 3D, allowing the turbulence to be correctly resolved, and taking care of the wing's deformation in 3D particularly the spanwise effects on the flow field.

The focus of this paper is twofold: firstly to perform a comparative steady CFD analysis between a wing equipped with a morphing TEF and seamless transition already deflected (i.e. statically morphed), and a wing with a conventional hinged flap and unsealed side-edge gaps. Secondly, an extension to the work investigating the unsteady aerodynamic effects of a morphing wing $([20,21])$ will be proposed in order to study a dynamically morphing TEF with a seamless transition (where the TEF deforms from a baseline position to a final deflection). A modified transition function will be used to model the transition between the static and morphing part of the wing. This method is then implemented in Ansys Fluent [22] in order deform the mesh along with the geometry. Finally, the aerodynamic performance of the unsteady morphing TEF is investigated, and results compared with the steady state results for the statically morphed wing configuration.

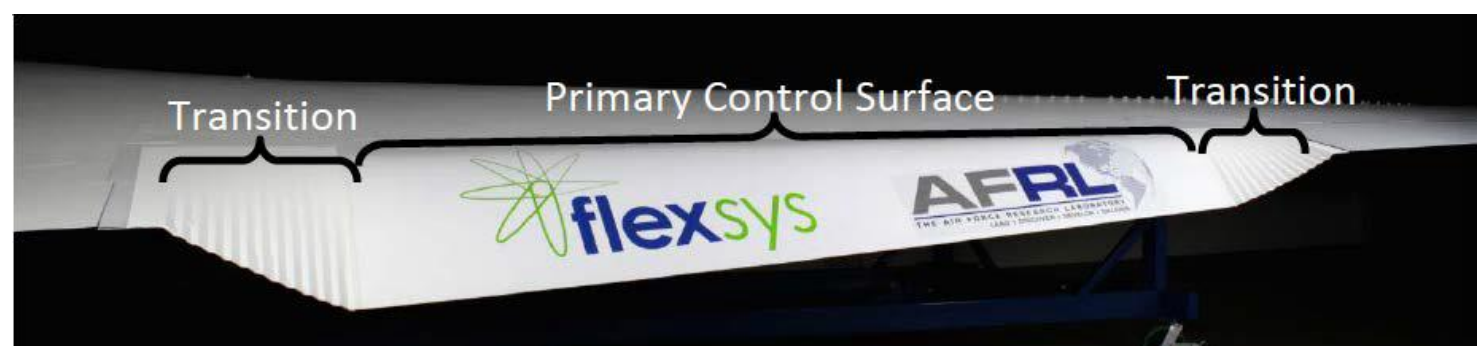

Fig.1 Morphing flap on Gulfstream III business jet, showing non-smooth transition surfaces [9].

\section{Problem definition}

Two cases are investigated in this work; first, a steady state CFD of statically morphed TEF is compared with a conventional hinged flap, whilst the second case will investigate a dynamically morphing TEF using a modified parametrization method. All configurations were studied at a Reynolds number based on the chord length of $\mathrm{Re}=0.62 \times 10^{6}$, and a Mach number of 0.115. A range of Angles of Attack (AoA) from $4^{\circ}$ to $8^{\circ}$ was considered for the steady analysis and the results at AoA $=6^{\circ}$ are presented for the dynamically morphing TEF.

\section{A. Steady RANS of a statically morphed TEF vs a hinged flap}

In order to study the differences in the aerodynamic behavior between a seamless transition flap with a sealed sideedge gaps and a conventional hinged flap, a rectangular NACA 0012 wing demonstrator with a chord $c=1 \mathrm{~m}$ and a span $S=1 \mathrm{~m}$ was investigated. The statically morphed TEF portion was set to be $50 \%$ of span and $25 \%$ allocated to each side transition starting from the side-walls. The same proportions were used for the wing with the hinged flap, where all side-edge gaps have a width of $1 \%$ of the chord $c$. Both wings were deflected to the same position, a vertical distance of $5 \%$ of the chord (approximately $14^{\circ}$ flap deflection angle). To gain further insight on how the flaps affect the performance, steady CFD was also performed for the baseline NACA 0012 wing and the baseline wing with $0^{\circ}$ flap deflection. Figure 2a summarizes all configurations studied while Fig. $2 \mathrm{~b}$ illustrates the 3D view of the morphed and hinged flap concepts. It is worth mentioning that a plain flap design for the hinged flap was chosen in order to provide similar size to the morphing TEF even though it is not the optimal aerodynamic design as opposed to built-in or split flaps for instance.

\section{B. Unsteady RANS of a dynamically morphing TEF}

The second part of the work will investigate the deformation motion of a wing fitted with a morphing TEF and a seamless side-edge transition. However, in order to model the deformation of the wing, it is important to define in time the mathematical formulae that analytically models such deformations. For the present problem, two parametrization methods are required. The TEF deformation is parametrized using the modified method [20] 
introduced in [17] and re-written in Eq. 1 and Eq.2. The unsteady camber distribution is added to the NACA 0012 thickness distribution in order to obtain the desired deformation.

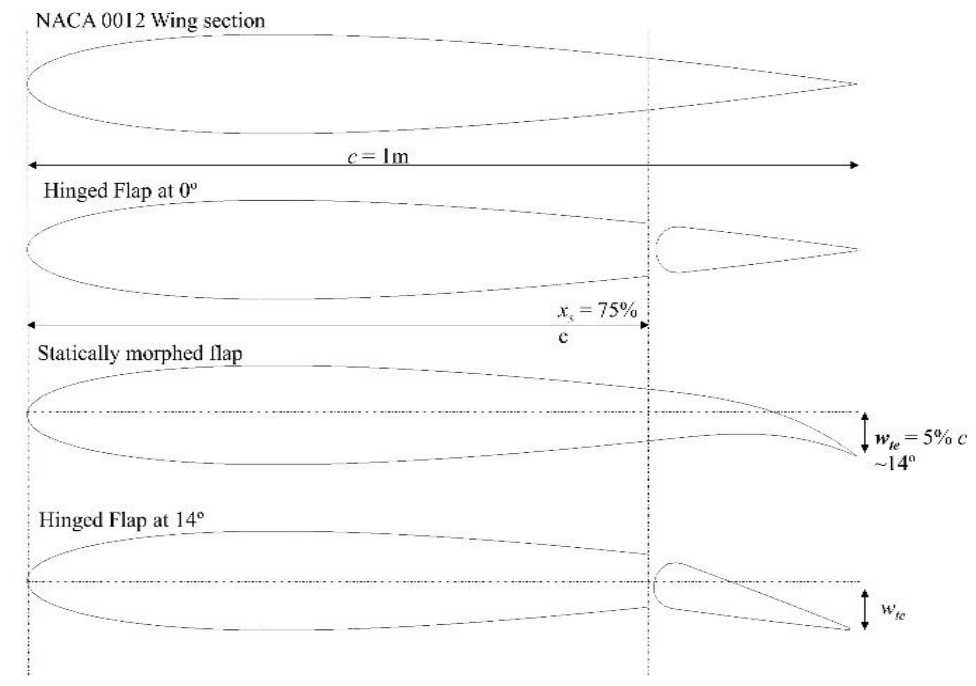

a) Mid-span slices showing the configurations studied and their dimensions.
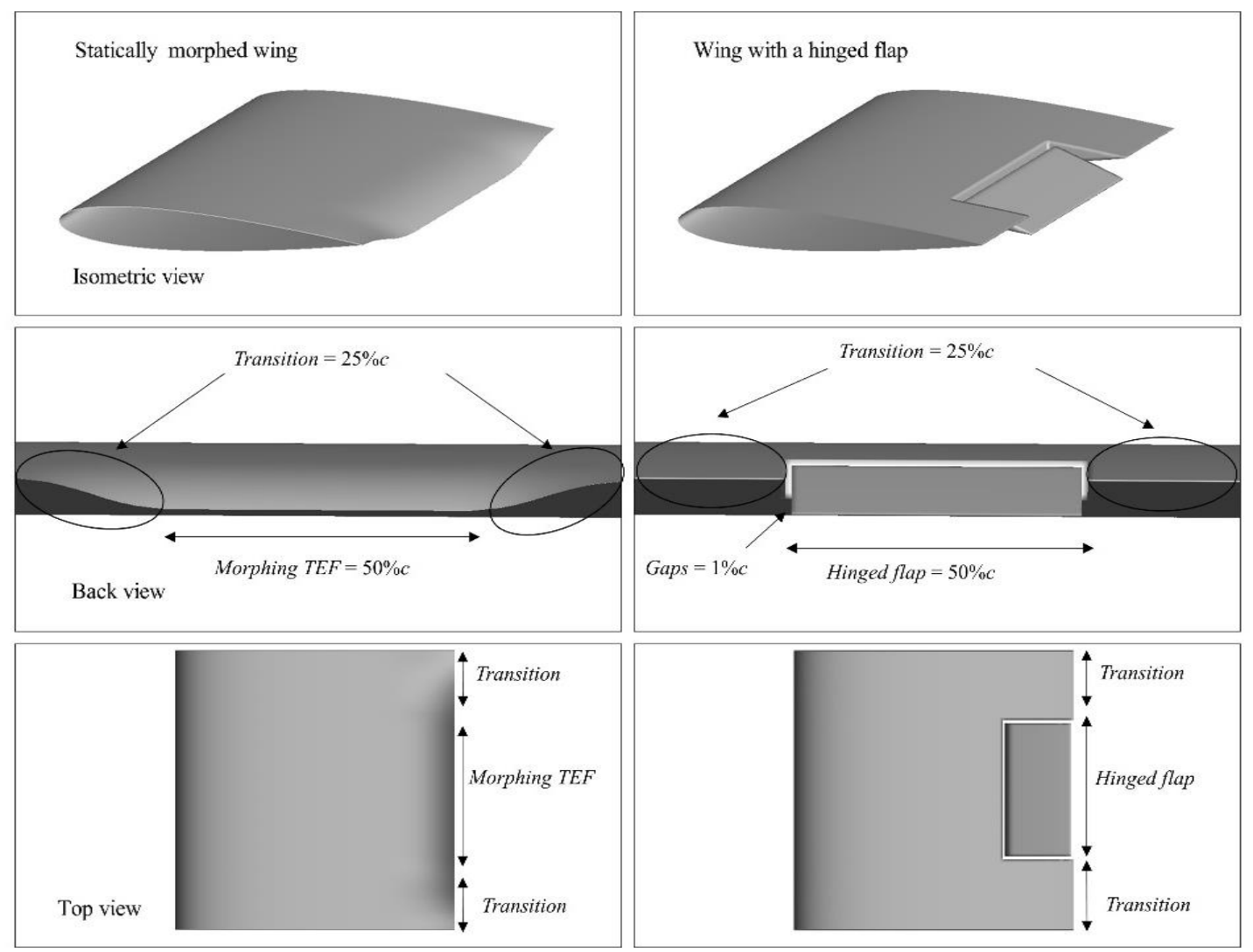

b) 3D models of the statically morphed wing and the hinged flap wing from various views.

Fig.2 Illustration of various configurations investigated. 


$$
y_{t}=\left(\frac{t h}{0.2}\right)\left(0.2969 \sqrt{\bar{x}}-0.1260 \bar{x}-0.3516 \bar{x}^{2}+0.2843 \bar{x}^{3}-0.1015 \bar{x}^{4}\right)
$$

$$
y_{c}=\left\{\begin{array}{l}
0 \quad, 0 \leq \bar{x}<x_{s} \text { and } 0 \leq t \leq \frac{T}{4} \\
\frac{-w_{t e} \sin \left(\frac{2 \pi t}{T}\right)\left(\bar{x}-x_{s}\right)^{3}}{\left(1-x_{s}\right)^{3}}, \bar{x} \geq x_{s} \text { and } 0 \leq t \leq \frac{T}{4} \\
\frac{-w_{t e}\left(\bar{x}-x_{s}\right)^{3}}{\left(1-x_{s}\right)^{3}}, \bar{x} \geq x_{s} \text { and } t>\frac{T}{4}
\end{array}\right.
$$

where $w_{t e}$ is the value of maximum deflection at the TE, $T$ is the morphing period and $x_{s}$ is the start location for the morphing (75\% for the subsequent studies).

Moving from 2D to 3D raises the issue of implementing an unsteady parametrization to model the side-edge transition between the morphing and non-morphing parts. Woods et al. [11] proposed a simple parametric formula (Eq.3) which yields a smooth continuous profile suitable for our application but viable only for the static cases.

$$
z_{t}=h \cdot \cos \left(\frac{\pi \bar{z}}{l}\right)-h
$$

where $z_{t}$ is the vertical TEF displacement for the transition part, $\bar{z}$ is the non-dimensional transition distance along the span and $h$ is the half-amplitude of the control surface deflection.

This method is modified as shown in Eq. 4 to reflect the unsteady deformation desired in our application and to introduce unsteadiness to the geometry.

$$
z_{t}=\left(h \cdot \cos \left(\frac{\pi \bar{z}}{l}\right)-h\right) \cdot \sin \left(\frac{2 \pi\left(t-t_{\text {start }}\right)}{T}\right)\left(\bar{x}-x_{s}\right)^{3}
$$

where $t$ is time and $T$ is the complete period of the TEF motion. At $t=t_{\text {start }}$ the morphing commences and the baseline is deflected until it reaches the maximum deflection value of $h$ thus simulating the deforming motion.

Figure $2 \mathrm{~b}$ (left) illustrates the final geometry resulting from the implementation of Eq. 2 and Eq. 4, from three point of views. The lower right figure clearly illustrates the smooth transition created by the function blending with the TE of the morphed flap.

\section{Computational setup}

\section{A. Steady RANS}

A steady state RANS study using the software package Ansys Fluent was conducted for the cases mentioned with a series of grids generated around each configuration. With a number of cells ranging between 1-1.5 $\mathrm{M}$ cells, and refinement regions placed around the wing and wake region, the farfield was placed at least 15 chords lengths away from the wings TE. A maximum near-wall first-layer grid resolution of $y^{+}<1$ was targeted. Hybrid grids (consisting of prism layer covering the boundary layer and tetrahedral elements outside) were favored for cases where the geometries had gaps or if the geometry was deforming. Hybrid meshes are faster to generate while keeping good mesh 
quality metrics. Furthermore, having tetrahedral elements offers the possibility to use re-meshing to remove skewed elements. Finally, a structured C-grid was used for the statically morphed wing giving the relative simplicity of the geometry. Figure 3 illustrates the computational domains used.

The pressure-velocity coupling is achieved using the coupled algorithm, the least squares cell based spatial discretization was used for all gradients. Moreover, the intermittency $k$ - $\omega$-SST model was used for turbulence closure; a second order upwind scheme was utilized for the momentum and turbulence equations discretization. In order to eliminate possible influence of wing tip vortices in this analysis, the wing was modelled as a semi-infinite wing where the width of the domain matches the span of the studied wing, and a zero shear stress boundary condition was imposed on the sidewalls of the domain. All simulations were run until the $C_{L}$ and $C_{D}$ statically converged and all the residuals dropped below $10^{-4}$.

\section{B. Unsteady RANS and dynamic meshing}

The unsteady RANS was performed using the baseline mesh generated for the NACA 0012 wing at AoA $=6^{\circ}$, it was initialized from converged steady state simulation results, and run until both $C_{L}$ and $C_{D}$ statically converged before engaging the dynamic meshing solver and starting the wing deformation.

In order to deform the mesh, diffusion-based smoothing was applied. Diffusion smoothing was chosen given its capability of better preserving mesh quality compared with other smoothing schemes [22] despite its higher computational cost compared with the spring-based smoothing for instance. In addition, re-meshing was used for cells having a skewness greater than 0.9. Figure 4 shows the mesh before and after the deformation of the TEF.

All the solver settings were similar to the steady analysis, additionally a $2^{\text {nd }}$ order transient discretization was used with a time step $\Delta t=10^{-4} \mathrm{~s}$ with 10 iterations per time step.

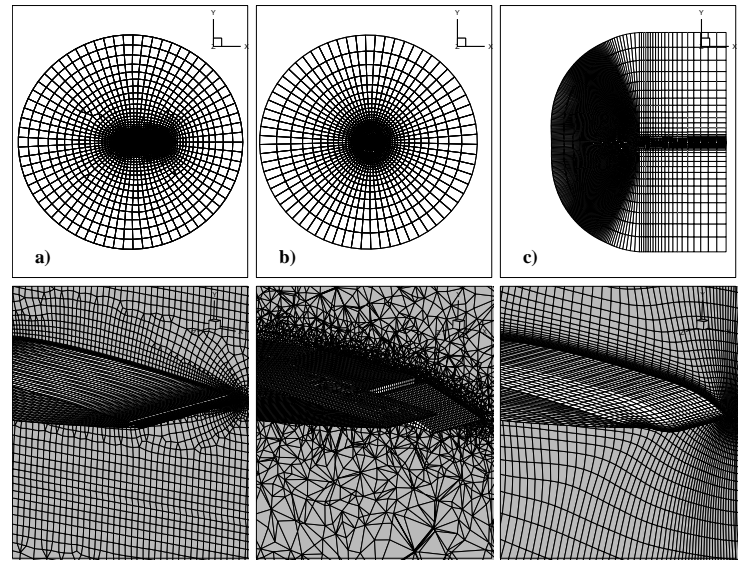

Fig.3 Computational domains used for the steady RANS and a close-up of the baseline NACA0012 wing, a) hinged flap wing $b$ ) and statically deformed meshes c).

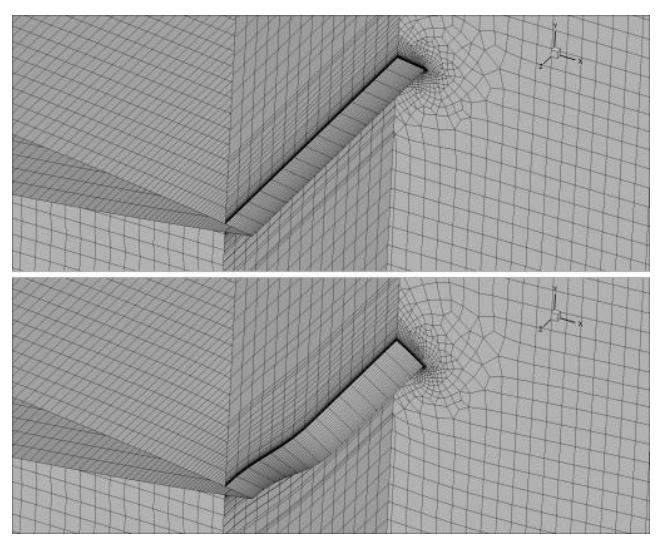

Fig. 4 Direct comparison between the baseline mesh (upper) and the mesh after the TEF deformation (lower).

\section{Results and Discussion}

\section{A. Baseline comparative study}

The lift and drag coefficients are plotted in Fig.5a, $5 \mathrm{~b}$ and $5 \mathrm{c}$ respectively, in addition to the aerodynamic efficiency of the four cases studied (NACA 0012 wing, morphed wing, wing with a hinged flap at $0^{\circ}$ and at $14^{\circ}$ ). Additionally, wind tunnel results of a NACA 0012 wing with side plates at similar Re number $\left(\sim 0.7 \times 10^{6}\right)$, are plotted for reference.

Both lift and drag results from the baseline NACA 0012 wing compare well to the experimental results, especially in the linear region of the flow, however at $\mathrm{AoA} 8^{\circ}$ discrepancies become higher exhibiting lower experimental values of $C_{D}$, which produces larger aerodynamic efficiency. This is expected as at the end of the linear region, the wing experiences more sepration which causes higher drag and lower lift comparing to the experimental test of a wing with 
side plates. The wing with a flap at $0^{\circ}$ (with the gaps) displays a similar behavior compared with the NACA 0012 wing, creating slightly lower levels of lift. However, the wing with the flap at $0^{\circ}$ consistently generates higher drag. This is due to the presence of the gaps; each gap width is $1 \%$, which reduces the total lifting area, causing a reduction in lift. Furthermore, the presence of the gaps gives rise to pressure leaks from the pressure to the suction side creating spikes in the pressure distribution in addition to extra recirculation regions effectively contributing in the profile drag.

\section{B. Statically morphed TEF vs hinged flap}

Comparing the aerodynamic performance of the wing equipped with the TEF and a seamless transition with the traditional hinged TEF give an insight into their behavior. When it comes to lift generation, the morphed wing unfailingly produces around $40 \%$ higher lift compared with the hinged flap configuration for similar or lower drag at AoA lower than $7^{\circ}$ giving an average of $45 \%$ higher aerodynamic efficiency. Yet at $8^{\circ}$ this trend seems to change as the morphed wing drag increases suddenly without any extra lift generated indicating that the wing may be approaching stall at angles around $8^{\circ}$ (stall angle for the NACA 0012 at this Re is $11^{\circ}$ [24]). Conversely, the hinged flap wing seems to maintain its efficiency at higher AoA which is in accordance with previous numerical and experimental results where it was found that a hinged flap preforms better at higher AoA compared with a morphed one $[20,21]$.

The large difference in the lift produced could be explained by the fact that the morphed wing has two side transition portions deflecting with the flap, creating additional deflection in the camber. This contributes to the extra lift, whereas the side edges of the hinged flap are static which does not contribute to lift as much as the seamless

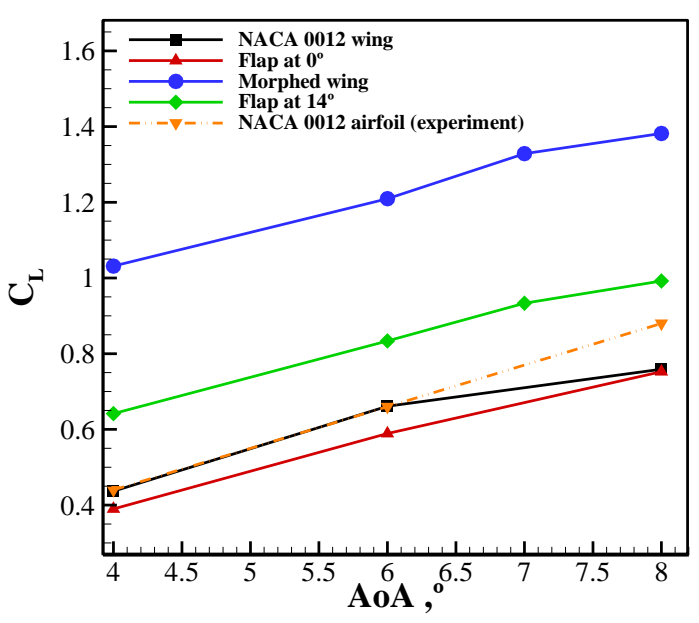

a) Lift Coefficient

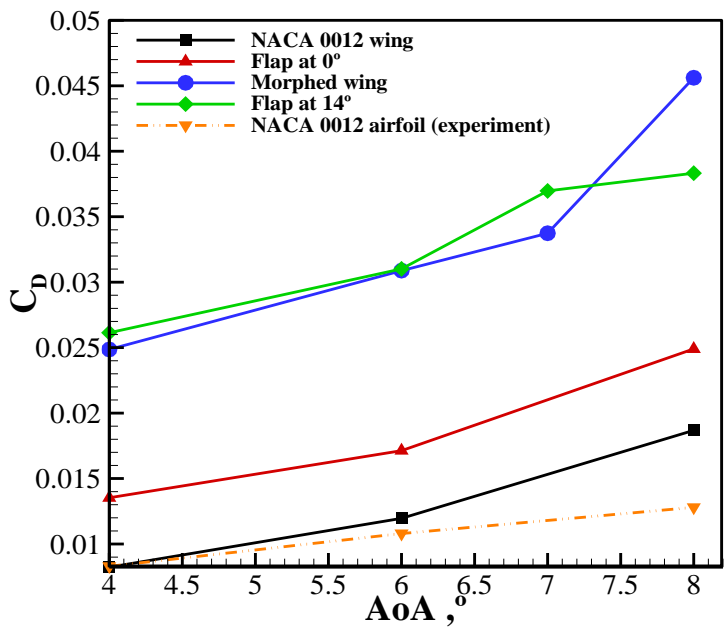

b) Drag Coefficient

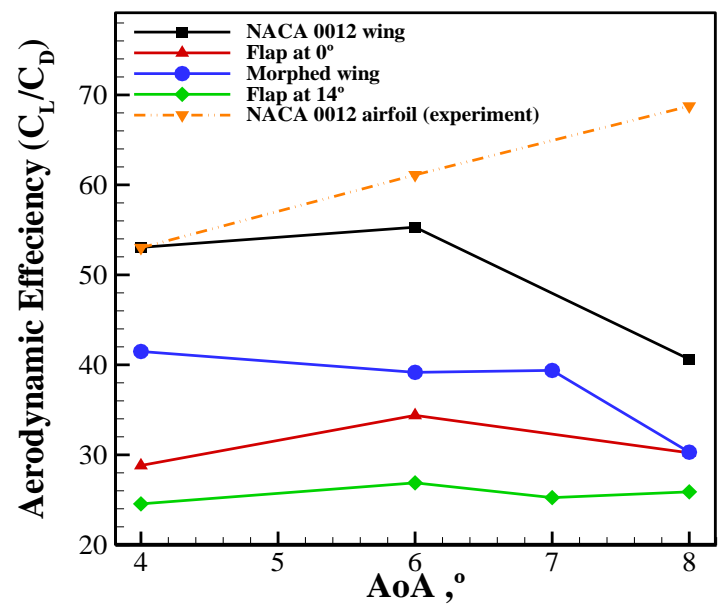

c) Aerodynamic efficiency

Fig.5 Comparative results for $C_{L}, C_{D}$ and the aerodynamic efficiency $\left(C_{L} / C_{D}\right)$ for the studied cases in addition to experimental results for the NACA 0012 airfoil from Sheldah et al. [24]. 
transition. Likewise, the presence of the gaps in the hinged flap wing induces extra recirculation areas and cavity flows between the gaps while reducing the effective lifting surface, which explains the lower aerodynamic efficiency observed for the hinged flap. Figure 6 illustrates the differences observed between the wing with the seamless transition and the one with a hinged flap. Recirculation areas around the gaps are clearly represented with velocity vectors. Moreover, a separation region on the pressure side of the flap seems to be constantly present, growing larger at higher AoA. In contrast, the velocity field distribution is smoother around the morphed wing, where the separation area seems to be larger on the sides than on the morphing flap.

Figure 7 gives in-depth insight into the behavior of the configurations studied by presenting the $C_{p}$ and $C_{f}$ (Fig.7a) distributions at various key locations on the wings, in addition to surface pressure contours, velocity contours and streamlines (Fig.7b) to inspect qualitatively and quantitatively the flow. At the location $z=0.251 \mathrm{~S}$ located just near the side edge gap, $C_{p}$ distribution comparison between the two wings shows a clear difference at the TEF region. The seamless transition has a much larger $C_{p}$ compared with the hinged flap wing, which results into a higher contribution by the seamless surface to lift, compared with the static side of the hinged flap wing that has a minimal contribution, which confirms that the seamless transition contributes more to the lift. The $C_{f}$ distribution at the same location shows the differences in the separation behavior, the morphed TEF wing keeps the flow attached longer whereas separation occurs earlier near the edge of the gaps, which is expected.

At the mid-plane location $(z=0.5 S)$ a direct comparison between the FishBAC morphed flap concept and a hinged flap can be made. The $C_{p}$ is larger around the entire morphed section thus creating more lift compared with the hinged flap case (Fig.7a). Two peaks are noticeable in the pressure distribution of the hinged flapped case near the location of the gaps, indicating the presence of the jet flow coming from the pressure side to the suction side through the gaps. This jet interaction with the separating flow from the main wing could have a flow control effect that could contribute to the consistent efficiency of the hinged flap wing at higher AoA. This behavior could be clearly detected from the $C_{f}$ plots as well where the separation is characterized by peak near the gaps. Moreover, the velocity streamlines around flap (Fig.7b) show streamlines from the suction side penetrating through the gaps and mixing with the separation region. In contrast, the morphed wing velocity streamline smoothly flow from the suction and pressure side and exhibit mixing with the separating shear layer.
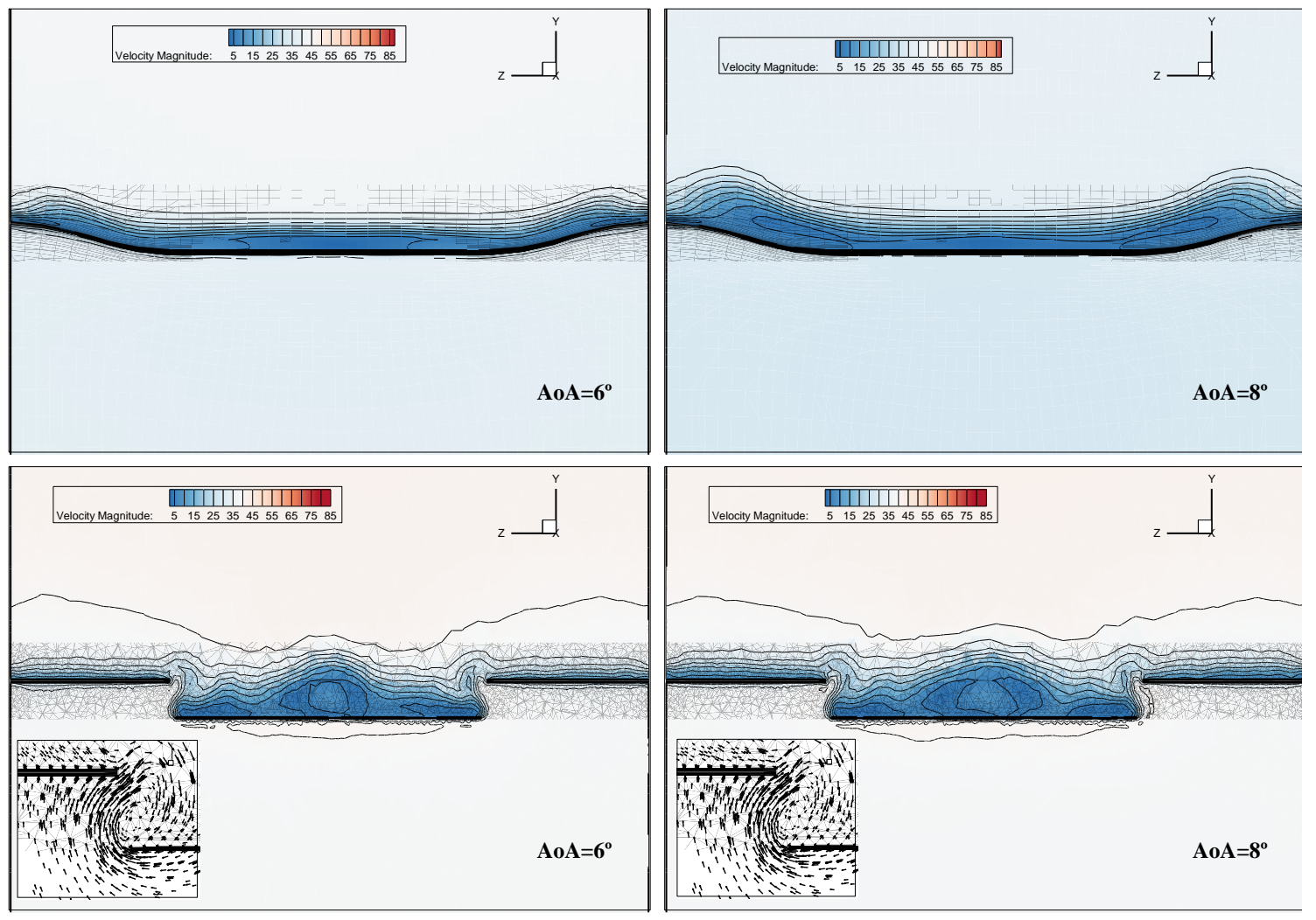

Fig. 6 Velocity contours and vectors of the wing with a morphed TEF flap (top) compared with the one with a hinged flap (bottom) on a plane placed at $\mathrm{x}=0.99 \mathrm{c}$ at $\mathrm{AoA}=6^{\circ}$ and $8^{\circ}$. 

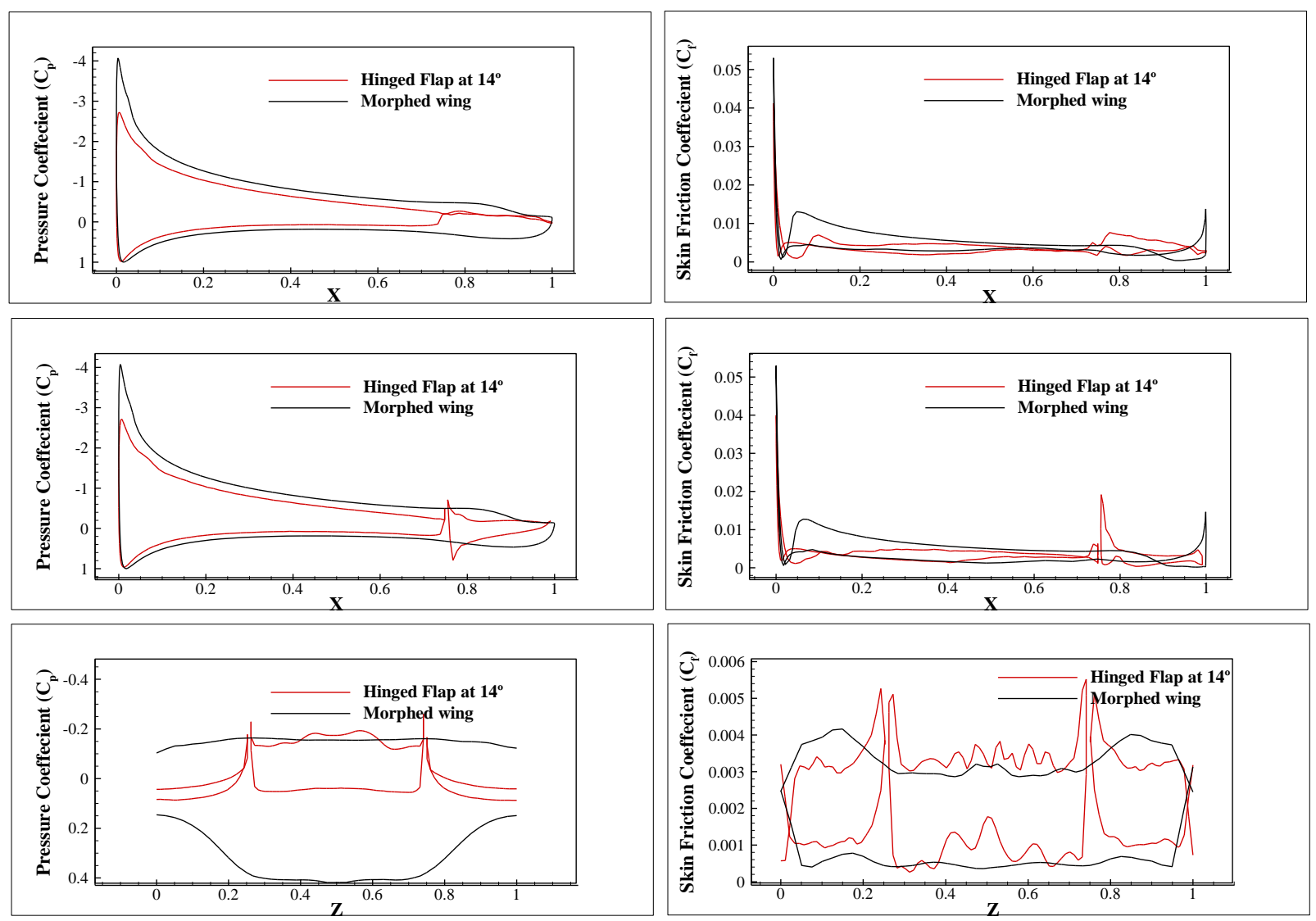

a) $C_{p}$ and $C_{f}$ at various plane locations at $A o A=6^{\circ}$.

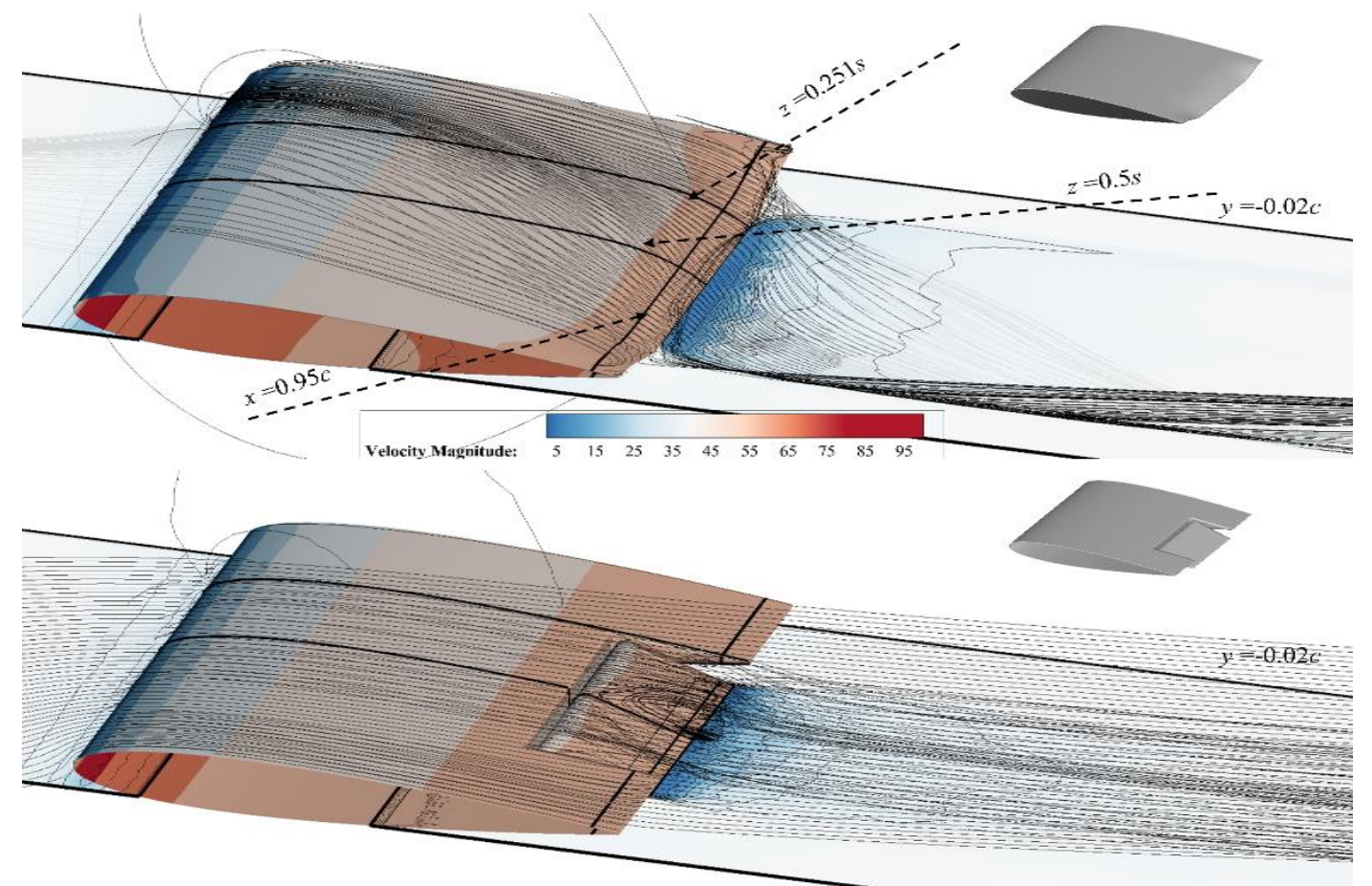

b) Surface pressure contours, velocity field and velocity streamlines.

Fig. 7 Quantitative and qualitative comparison between the wing with a morphing TEF and seamless transition and the wing with a hinged flap. 
Finally, spanwise $C_{p}$ and $C_{f}$ distributions at location $x=0.95 c$ are presented in Fig. 6 as well. This graph gives a clear illustration of the differences a seamless transition and an unsealed side-edge flap could produce. The morphed flap $C_{p}$ distribution is continuous and overall larger than the hinged flap case where the static side edge has a smaller $C_{p}$ distribution showing the peaks produced as a result of the unsealed gaps. The $C_{f}$ distribution is overall more similar in size, confirming the similar $C_{D}$ results obtained, however the flow on the flap appears to be more turbulent, with strong instabilities present at the gaps which are the main contributors in profile drag and main noise source whereas the seamless morphed wing effectively eliminates those sources.

\section{Unsteady RANS of a dynamically morphing TEF}

Figure 8 shows the results obtained for the dynamically morphing TEF, the morphing start time was set to be $t_{\text {start }}=0.5 \mathrm{~s}$ to give time for wing unsteady quantities to statically converge before starting the morphing process which lasts another $0.5 \mathrm{~s}$ and stops at $t=1 \mathrm{~s}$. The lift and drag coefficient increase in a quasi-linear fashion throughout the morphing process, with forces oscillating about a mean flow, giving an indication of the presence of continuous vortex shedding and Laminar Boundary Layer (LBL) instabilities. Shortly before the morphing stops, a peak in $C_{D}$ is observed before the values settles down. Comparing the unsteady and steady results, the difference in lift is about $2 \%$, however the drag coefficient predicted with the unsteady analysis is about $20 \%$ lower showing that the morphed wing is even more efficient given the unsteady results tends to resolve the flow details better producing better accuracy predictions. When taking into account the entire morphing period the average lift goes down by $11 \%$ while the average drag increases by $3 \%$. These predictions are in fact more realistic comparing to the statically morphed wing, as in real life scenarios, the flap will be deployed dynamically which gives raise to unsteady phenomena that could influence the performance such as the sudden peak in drag observed before the final TEF position.

For more insight into the unsteady morphing process, the $C_{p}$ and $C_{f}$ distribution are presented in Fig.9 and Fig.10 various locations and instances from the start of the morphing until after the morphing ends. The $C_{p}$ diagrams clearly illustrate the effect that the change in camber has on the pressure distribution. The $C_{p}$ distribution gets larger the more the TEF is morphed creating higher lift, in addition the LBL instabilities were not present at the start of the morphing, but when the morphing is ongoing, the LBL instabilities appears to get stronger around the pressure side and near the LE of the wing. The effect of varying the camber are also present in the $C_{f}$ distribution, the flow appears to be laminar for the baseline at the beginning of morphing however the more the flap is deflected the more turbulent the boundary layer gets. At $t=1 s$ and $1.4 s$ the transition to turbulent BL is clearly seen in the peaks, and it appears that it gets closer to the LE the more the main flap is deflected.

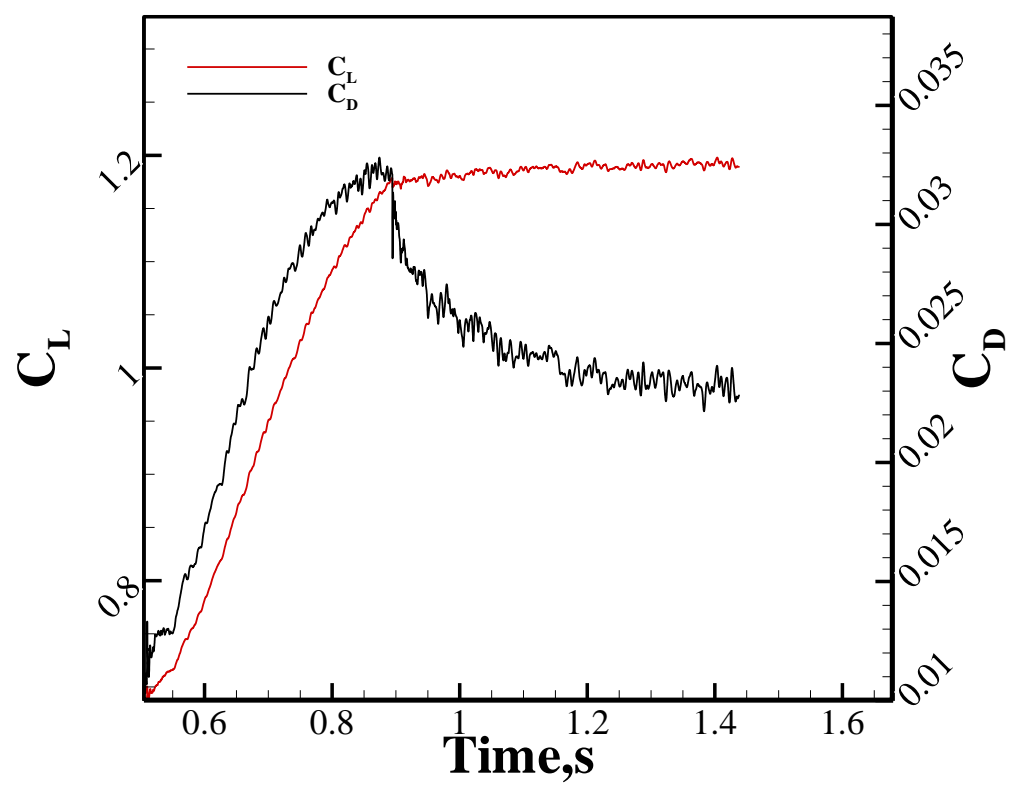

Fig.8 Instantaneous $C_{L}$ and $C_{D}$ for the dynamically morphing TEF at $A 0 A=6^{\circ}$. 

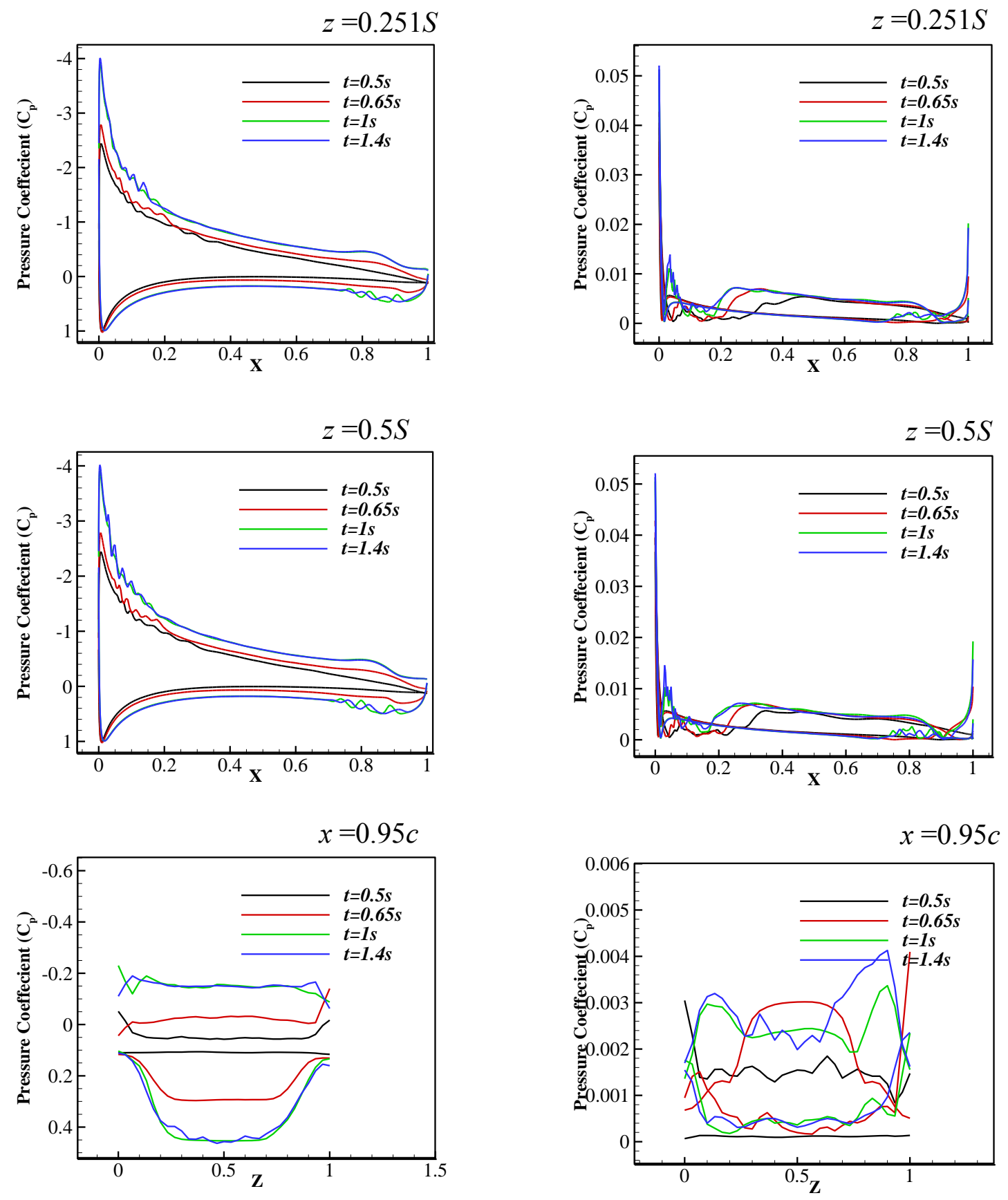

Fig.9 $C_{p}$ distribution at various instances for the dynamically morphing TEF at $\mathrm{AoA}=6^{\circ}$.

Fig.10 $C_{f}$ distribution at various instances for the dynamically morphing TEF at $\mathrm{AoA}=6^{\circ}$.

Finally, the spanwise distribution of $C_{f}$ indicates the presence of a growing recirculation area at the TE with the largest regions present at the tips making the distribution asymmetrical (given the oscillatory behavior of the instabilities). Fig.11 further illustrates this behavior by showing the Q-criterion iso-surfaces colored by the velocity magnitude for the initial NACA 0012 wing prior to morphing and after morphing at $t=1.4 \mathrm{~s}$. The figure clearly shows the increase of the size and strength of boundary layer the instabilities at the LE and the TE vortices when the wing is morphed from the baseline to the maximum deflection. 

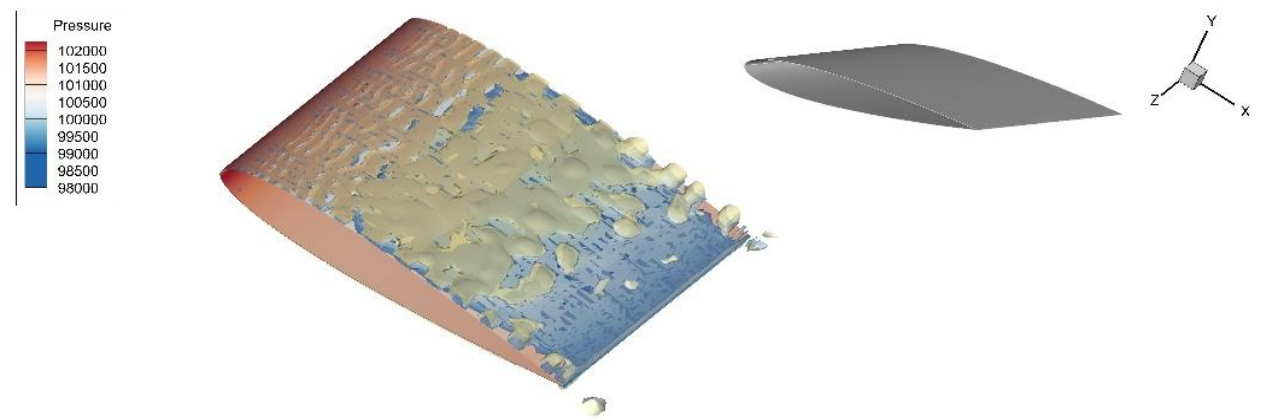

$t=0.5 \mathrm{~s}$

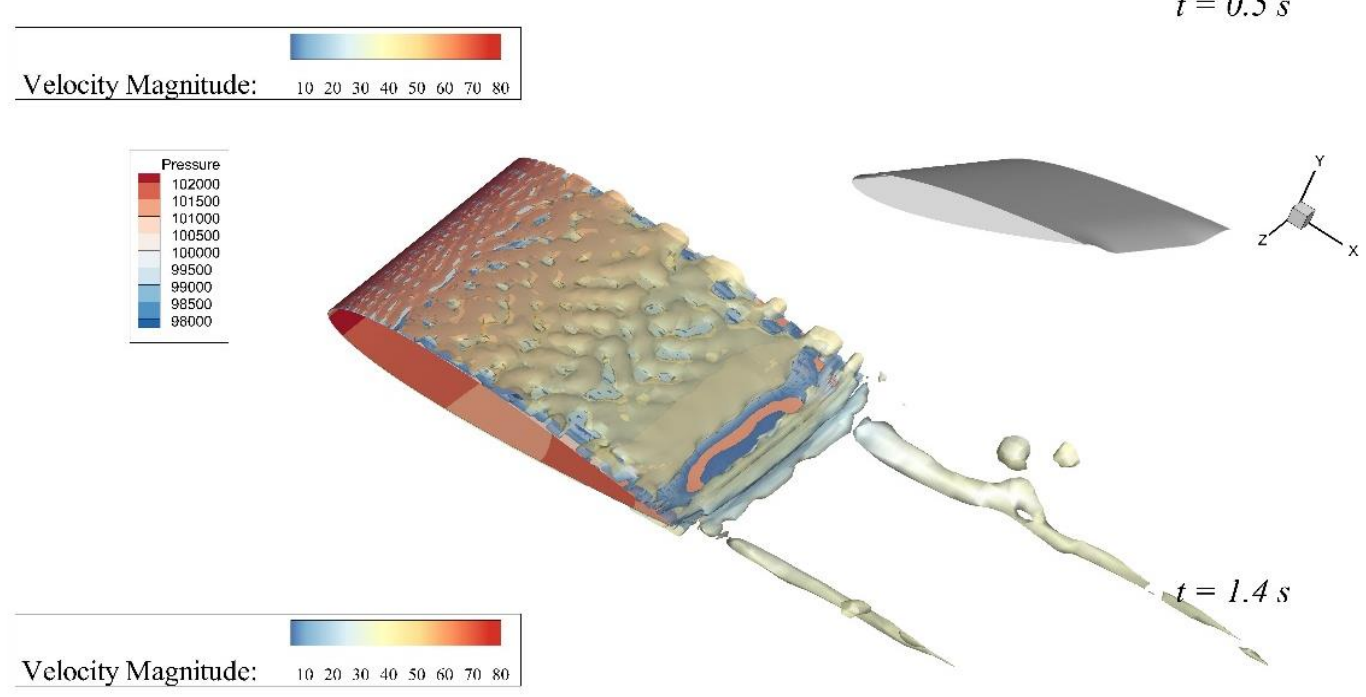

Fig. $11 \mathrm{Q}$ criterion iso-surfaces colored by velocity magnitude for the dynamically morphing TEF at $\mathrm{AoA}=6^{\circ} t=0.5 \mathrm{~s}$ and $1.4 \mathrm{~s}$.

\section{Conclusion and Future Work}

A 3D steady RANS analysis of a statically morphed TEF with seamless transition was performed and results compared with both the baseline wing and with a traditional hinged flap configuration at a $\mathrm{Re}=0.62 \times 10^{6}$ for a range of angles of attack from 0 to $8^{\circ}$. In order to perform the unsteady analysis of dynamically morphing TEF with seamless transition, a parametrization method was modified and implemented in a UDF in order to drive the dynamic meshing in Ansys Fluent.

It was found that the baseline NACA 0012 wing produced results comparable with published experimental data for the NACA 0012 airfoil. Interestingly, the presence of the gap even at $0^{\circ}$ flap deflection angle was found to have negative effects on the aerodynamic performance. Moreover, at low AoA the morphed wing was found to have up to $40 \%$ better aerodynamic efficiency due to the seamless transition contribution to the lift in comparison with the static part of the hinged flap wing. Nevertheless, the morphed wing showed deteriorating performance at higher AoA whereas the hinged flap behaved consistently which is in agreement with previous research.

Finally, the parametrization method was successfully implemented and unsteady flow analysis at $A o A=6^{\circ}$ was performed offering the possibility to include the deformation motion in the modelling of such morphing configurations. Results showed that due to unsteady effects the average aerodynamic efficiency for the entire morphing time is lower than the one for the statically morphed TEF proving the need to take into account the morphing motion while in the design process. 
In the future, the developed framework will be used to conduct high fidelity parametric studies of morphing wings configurations at different deflection angles and frequencies. In addition, this framework will allow the exploration of $3 \mathrm{D}$ harmonic forcing in both the streamwise and spanwise directions.

\section{ACKNOWLEDGMENTS}

The first author gratefully acknowledges the studentship funding received from the Engineering Modelling and Simulation Research Group, University of the West of England, Bristol, UK.

\section{References}

[1] European Commission., "Flightpath 2050: Europe's vision for aviation," ISBN 978-92-79-19724-6, p. 28, 2011.

[2] Weisshaar, T. A. "Morphing aircraft technology-new shapes for aircraft design Multifunctional Structures/Integration of Sensors and Antennas." In Meeting Proceedings RTOMP-AVT-141, Overview 1. Neuilly-sur-Seine, France: RTO, p. O1. 2006.

[3] Barbarino, S., Bilgen, O., Ajaj, R. M., Friswell, M. I., and Inman, D. J. "A Review of Morphing Aircraft," Journal of intelligent material systems and structures, vol. 22, no. 9, pp. 823-877, 2011. doi: 10.1177/1045389X11414084.

[4] Nguyen, N "Project Elastically Shaped Future Air Vehicle Concept," NASA Innovation Fund Award, 2010.

[5] Urnes, J., Nguyen, N., Ippolito, C., Totah, J., Trinh, K., and Ting, E. “A Mission Adaptive Variable Camber Flap Control System to Optimize High Lift and Cruise Lift to Drag Ratios of Future N+3 Transport Aircraft," in Aerospace Sciences Meetings ,AIAA-2013-214, , 2013, no. 650, pp. 1-24. doi: 10.2514/6.2013-214.

[6] Macaraeg, M. G. "Fundamental investigations of airframe noise," in 4th AIAA/CEAS Aeroacoustics Conference, 1998, pp. 123-132. doi: 10.2514/6.1998-2224

[7] Khorrami, M.R., Lockard, D.P., Moore, J.B., Su, J., Turner, T.L., Lin, J.C., Taminger, K.M., Kahng, S.K. and Verden, S.A "Elastically deformable side-edge link for trailing-edge flap aeroacoustic noise reduction," atent No. US8695925B2. Issued Apr. 15, 2014, US8695925 B2, 15-Apr-2014.

[8] Kota, S., Osborn, R., Ervin, G., Maric, D., Flick, P., and Paul, D. "Mission adaptive compliant wing-design, fabrication and flight test," in RTO Applied Vehicle Technology Panel (AVT) Symposium, 2009.

[9] Kota, S., Flick, P., and Collier, F. "Flight Testing of FlexFloil "M Adaptive Compliant Trailing Edge," 54th AIAA Aerosp. Sci. Meet., no. January, AIAA 2016-0036, pp. 1-13, 2016. doi:10.2514/6.2016-0036.

[10] NASA, "Hear This: 30 Percent Less Noise," $2017 . \quad$ [Online]. Available: https://www.nasa.gov/centers/armstrong/feature/ACTE_30_percent_less_noise.html.

[11] Woods, B. K., Parsons, L., Coles, A. B., Fincham, J. H., and Friswell, M. I. "Morphing elastically lofted transition for active camber control surfaces," Aerosp. Sci. Technol., vol. 55, pp. 439-448, Aug. 2016.doi: 10.1016/j.ast.2016.06.017.

[12] Woods, B. K. S., and Friswell, M. I. "Preliminary investigation of a fishbone active camber concept," in Proceedings of the ASME 2012 conference on smart materials, adaptive structures and intelligent systems, 2012, pp. 555-563.

[13] Molinari, G., Quack, M., Dmitriev, V., Morari, M., Jenny, P., and Ermanni, P. “Aero-Structural Optimization of Morphing Airfoils for Adaptive Wings," Journal of Intelligent Material Systems and Structures, vol. 22, no. 10, pp. 1075-1089, 2011. doi: 10.1177/1045389X11414089.

[14] Obradovic, B. and Subbarao, K. "Modeling and Simulation of Morphing Wing Aircraft," Morphing Aerosp. Veh. Struct., vol. 48, no. 2, pp. 87-125, 2012.doi: 10.1002/9781119964032.ch5.

[15] Lyu, Z., and Martins, J. R. “Aerodynamic Shape Optimization of an Adaptive Morphing Trailing-Edge Wing,” Journal of Aircraft,AIAA., vol. 52, no. 6, pp. 1951-1970, 2015.doi: 10.2514/1.C033116

[16] Ai, Q., Kamliya Jawahar, H., and Azarpeyvand, M "Experimental investigation of aerodynamic performance of airfoils fitted with morphing trailing edges," 54th AIAA Aerosp. Sci. Meet., no. January, 2016.

[17] Woods, B. K., Fincham, J. H., \& Friswell, M. I. "Aerodynamic Modelling of the Fish Bone Active Camber Morphing Concept Aerodynamic Modelling of the Fish Bone Active Camber Morphing Concept," Journal of Intelligent Material Systems and Structures. SAGE Publications, 25(7), pp. 772-785. July, 2014. doi: 
$10.1177 / 1045389 X 14521700$.

[18] Kamliya Jawahar, H., Ai, Q., \& Azarpeyvand, M. "Experimental and numerical investigation of aerodynamic performance of airfoils fitted with morphing trailing-edges," 23rd AIAA/CEAS Aeroacoustics Conf. 2017, AIAA 2017-3371. June, 2017.doi: 10.2514/6.2017-3371

[19] Ninian, D. and Dakka, S.M., "Design, Development and Testing of Shape Shifting Wing Model," Aerospace, vol. 4, no. 4, p. 52, 2017.doi: 10.3390/aerospace4040052

[20] Abdessemed, C., Yao, Y., Bouferrouk, A., \& Narayan, P. "Morphing airfoils unsteady flow analysis using dynamic meshing," International Journal of Numerical Methods for Heat \& Fluid Flow, vol. 23, no. 5, 2017.doi: 10.1108/HFF-06-2017-0261

[21] Abdessemed, C., Yao, Y., Bouferrouk, A., \& Narayan, P. "Unsteady parametrization of a morphing wing design for improved aerodynamic performance," 52nd 3AF International Conference on Applied Aerodynamics, Lyon, France, 27-29 March 2017.

[22] ANSYS, (2018) "Release 18.2, Help System.” ANSYS Fluent Theory Guide. ANSYS, Inc,2018.

[23] Abdessemed, C., Yao, Y., Bouferrouk, A., \& Narayan, P "Aeroacoustic Investigation of a Harmonically Morphing Trailing Edge Flap," 53rd 3AF International Conference on Applied Aerodynamics, Salon de Provence, France, 26 - 28 March 2018.

[24] Sheldahl, R. E., \& Klimas, P. C. “Aerodynamic characteristics of seven symmetrical airfoil sections through 180-degree angle of attack for use in aerodynamic analysis of vertical axis wind turbines," Sandia National Labs., Albuquerque, NM (USA), 1981.

[25] Beaverstock, C. S., Woods, B. K. S., Fincham, J. H. S. M., \& Friswell, M. I. "Performance Comparison between Optimised Camber and Span for a Morphing Wing," Aerospace , vol. 2, no. 3. 2015.doi: 10.3390/aerospace2030524 